\title{
Highly Efficient Wavelength Conversion of $Q$-Switched Nd:YAG Lasers Into a Red Wavelength
}

\author{
Ziya Gürkan Figen, Ateş Yalabik, and Orhan Aytür, Senior Member, IEEE
}

\begin{abstract}
We report a nanosecond upconversion optical parametric oscillator which can convert the output of a Nd:YAG laser at 1064-nm wavelength into $627 \mathrm{~nm}$ with high efficiency. This device is based on a single $\mathrm{KTiOAsO}_{4}$ crystal that is simultaneously phase matched for optical parametric generation and sum-frequency generation. Pumped at a wavelength of $1064 \mathrm{~nm}$ by a $Q$-switched Nd:YAG laser in a double-pass configuration, this device produces 14.2-ns-long 5.8-mJ red pulses at a wavelength of $627 \mathrm{~nm}$ with $29 \%$ energy conversion efficiency. In addition, we developed a theoretical model for this device, whose predictions agree reasonably well with our experimental results. It is possible to scale this device for use with higher energy pump lasers and to shape it into a physically small semi-monolithic configuration.
\end{abstract}

Index Terms-Frequency conversion, Neodymium:YAG lasers, nonlinear optics, optical parametric oscillators, $Q$-switched lasers.

\section{INTRODUCTION}

$\mathbf{C}$ OHERENT radiation in the red part of the spectrum has important applications in several areas including laser projection display technologies, holography, biomedical systems, and as an intermediate wavelength for conversion to other wavelengths of interest. Nonlinear wavelength conversion of solid-state lasers based on neodymium (Nd)-doped laser crystals to red wavelengths has been widely utilized for red beam generation. In particular, Nd:YAG [1]-[3], Nd:YLF [4], [5], $\mathrm{Nd}: \mathrm{YVO}_{4}$ [6], [7] and $\mathrm{Nd}: \mathrm{GdVO}_{4}$ [8], [9] lasers operating at around $1.3 \mu \mathrm{m}$ have been converted into red wavelengths using either extracavity or intracavity doubling schemes in the continuous-wave (cw), nanosecond, or picosecond regimes. Red beam generating optical parametric oscillators (OPOs) pumped by various harmonics of Nd:YAG lasers operating at 1064 $\mathrm{nm}$ were reported in the nanosecond pulsed regime [10]-[14]. However, the overall 1064-nm-to-visible energy conversion efficiencies of these systems are typically below $10 \%$. More recently, nanosecond OPOs using periodically poled crystals that are pumped by the second-harmonic of 1064-nm Nd:YAG lasers were demonstrated to yield larger conversion efficiencies for red beam generation [15], [16]. However, output energies of these devices were limited due to the damage threshold of the OPO crystal, a general problem resulting from aperture-size constraints in poled crystals.

Manuscript received January 8, 2006; revised March 8, 2006.

The authors are with the Department of Electrical and Electronics Engineering, Bilkent University, TR-06800 Ankara, Turkey (e-mail: gurkan@ee.bilkent.edu.tr; gurkanf@iltaren.tubitak.gov.trayalabik@gmail.com; aytur@ee.bilkent.edu.tr ).

Digital Object Identifier 10.1109/JQE.2006.876711

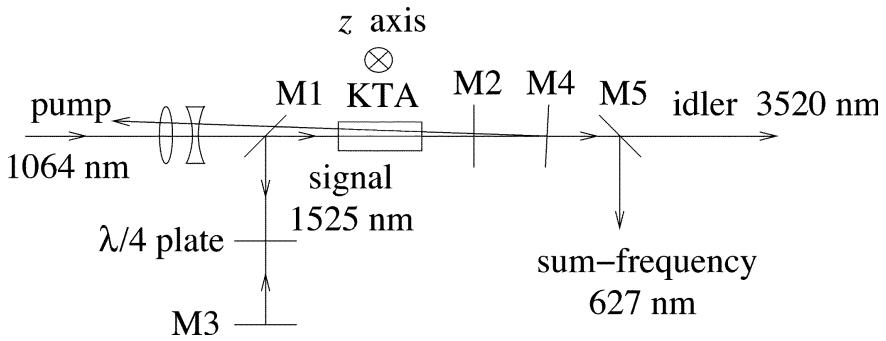

Fig. 1. Experimental setup of the SF-OPO with double-pass pumping configuration.

Combining two steps of frequency conversion within the same nonlinear crystal using simultaneous phase matching facilitates efficient frequency conversion to wavelengths that cannot be reached via a single nonlinear process [17]-[26]. In particular, both femtosecond and cw Ti:sapphire laser beams have been upconverted to visible wavelengths by combining sum-frequency generation (SFG) [20], [21] or second-harmonic generation [21], [23], [24] with optical parametric oscillation. We recently reported a sum-frequency generating OPO (SF-OPO) that extends this approach to the nanosecond regime [26].

In this paper, we report an SF-OPO in which the 1064-nm pump beam from a $Q$-switched Nd:YAG laser makes a doublepass through a $\mathrm{KTiOAsO}_{4}$ (KTA) crystal that is simultaneously phase-matched for both parametric generation and SFG. This device operates at room temperature and generates red output pulses at $627 \mathrm{~nm}$ with upto $29 \%$ 1064-627-nm energy conversion efficiency. This SF-OPO can be shaped into a small-size, semi-monolithic module to be placed in front of a Nd:YAG laser for highly efficient conversion into a red wavelength.

In addition, we report a theoretical model for our device and compare its predictions with the experimental results. Our model incorporates both temporal and spatial profiles of the beams, and takes diffraction into account.

\section{EXPERIMENTAL CONFIGURATION}

Our experimental setup is shown in Fig. 1. The pump source is a 4-Hz flash-lamp-pumped $Q$-switched Nd:YAG laser operating at $1064 \mathrm{~nm}$, generating 32-mJ pulses of 17.8-ns duration [fullwidth at half-maximum (FWHM)]. The telescope lenses reduce the diameter of the pump beam that has a Gaussian-like spatial profile almost threefold resulting in a 1.3-mm-diameter beam $\left(1 / e^{2}\right.$ intensity point) with a divergence of $3 \mathrm{mrad}$.

The SF-OPO is based on a 20-mm-long KTA crystal which has antireflection coatings for the pump and the signal wave- 
lengths on both surfaces and the propagation is along the $\theta=$ $90^{\circ}$ and $\phi=30.8^{\circ}$ direction of the crystal. The $4.8-\mathrm{cm}$-long L-shaped cavity is made up of three flat mirrors, M1, M2, and M3, which are all high reflectors at the signal wavelength of $1525 \mathrm{~nm}$. The pump beam entering the cavity through M1 and exiting through M2 makes a second-pass through the crystal as it returns into the cavity upon a reflection from the high-reflector mirror M4 placed about $3 \mathrm{~mm}$ away from M2. M4 is slightly tilted to prevent the back-reflection from coupling into the pump laser cavity by providing an angle of $0.2^{\circ}$ between the directions of the incident and return pump beams in the crystal. Both M1 and M2 are high transmitters at $1064 \mathrm{~nm}$. When pumped above threshold, a $p$-polarized (horizontal, fast axis) intracavity signal beam at $1525 \mathrm{~nm}$ and an $s$-polarized (vertical, slow axis, $z$ axis) idler beam at $3520 \mathrm{~nm}$ are generated from a $p$-polarized pump beam through parametric generation employing a type-II birefringent phase-matching geometry. An intracavity $\lambda / 4$ plate whose surfaces are antireflection coated at $1525 \mathrm{~nm}$ acts as a polarization rotator to couple a portion of the signal beam to $s$-polarization. Simultaneously phase-matched SFG results in the $s$-polarized component of the signal to be summed with the pump beam to produce a $p$-polarized sum-frequency beam at $627 \mathrm{~nm}$, again in a type-II polarization geometry. This red beam exits the cavity through M2 and is separated from the idler by the highly reflecting dichroic mirror M5. The red beam experiences a total loss of $22 \%$ in going through the second KTA surface, M2, and M4 (8\% each) because these surfaces were not antireflection coated for $627 \mathrm{~nm}$. The idler beam is totally absorbed in M2, M4, and M5, which are made from BK7 glass.

We have also operated the device in a single-pass pumping configuration where $\mathrm{M} 4$ is replaced with a $45^{\circ}$ dichroic mirror, which separates the pump from the sum-frequency. This dichroic mirror is a high reflector at the pump wavelength and has a transmission of $96 \%$ at $627 \mathrm{~nm}$. In this case, only a small amount of idler, $0.3 \mathrm{~mJ}$ at the highest input pulse energy, is measured after the mirrors external to the cavity.

For the double-pass and single-pass configurations, we calculated the peak intensities of the incident pump beam at the crystal input to be $140 \mathrm{MW} / \mathrm{cm}^{2}$ for a maximum pulse energy of $20 \mathrm{~mJ}$ and $210 \mathrm{MW} / \mathrm{cm}^{2}$ for a maximum pulse energy of $30 \mathrm{~mJ}$, respectively. At these incident pump levels, the corresponding intracavity signal pulse energies, whose relative values are monitored using a photo-detector placed behind M3, are similar in value. The peak pump intensities are kept below these values to avoid the damage of the surface coatings on the KTA crystal. The damage threshold of these coatings is specified to be $500 \mathrm{MW} / \mathrm{cm}^{2}$ for a $20-\mathrm{ns}$ pulse at $1064 \mathrm{~nm}$.

Our KTA crystal is cut along the $\theta=90^{\circ}$ and $\phi=32.6^{\circ}$ direction. However, experimentally we determined that simultaneous phase matching at our wavelengths occurs at $\theta=90^{\circ}$ and $\phi=30.8^{\circ}$, requiring a corresponding tilt. This propagation angle is quite close to the simultaneous phase-matching angle reported in [26] that was experimentally determined for a different KTA crystal and the angle calculated using the Sellmeier coefficients given in [27] for parametric generation and those given in [28] for SFG. The walk-off angles associated with the beams polarized along the fast axis are relatively small, with the maximum value being $0.15^{\circ}$ for the sum-frequency beam, whereas the beams polarized along the slow axis experience no walk-off.

\section{THEORETICAL MODEL}

The plane-wave analysis of SF-OPOs was previously reported in the continuous-wave regime [29]. However, evolutions of the temporal and spatial profiles of the beams have to be taken into account in an accurate model of a nanosecond SF-OPO. A numerical model which includes temporal profiles of the beams as well as diffraction, birefringent walk-off, and pump depletion was reported for nanosecond OPOs [30]. Here, we extend this model to nanosecond SF-OPOs.

In our model, a Gaussian pulse fitted to the temporal profile of the pump laser is discretized with a series of time slices separated by the round-trip time for the SF-OPO cavity. The time-integrated transverse profile of the pump beam measured at the input face of the crystal by a charge-coupled device (CCD) camera is also discretized to a transverse grid. Since the pump beam is collimated, the phase-fronts of the input pump beam are assumed to be flat at the input face of the crystal. For each time slice, the spatial profile of the pump is propagated through the nonlinear crystal. Propagation of all the interacting beams in the nonlinear crystal and in free space are handled using the Fourier transforms of the fields in the transverse dimension. We keep track of the phases and amplitudes of the depleted pump, $p$-polarized and $s$-polarized signal, idler, and sum-frequency fields over their transverse profiles at each time slice. The $p$-polarized signal spatial profile is updated at the input face of the crystal after one cavity round-trip, with residual cavity losses and the loss due to coupling into the $s$-polarized signal taken into account. This procedure is repeated for the duration of the pump pulse. We assume that the cavity is singly resonant for the signal, the idler and sum-frequency beams at the crystal input surface have zero amplitude and neglect residual reflections of all beams from the optical surfaces. The $s$-polarized signal is assumed to be completely depleted by SFG following a pass through the nonlinear crystal in the forward-propagation direction. In this model, we also assume narrow-bandwidth operation [30]. However, in the experiment, the SF-OPO produces multimode output since the pump laser is not injection-seeded. Two numerical approaches to modelling multimode OPOs have been reported [31], [32]. However, incorporating diffraction into these models results in prohibitively high computational costs. Nevertheless, we achieve reasonably good agreement between the predictions of our narrow-band model and our experimental results.

The simultaneous phase matching polarization geometry of our device belongs to class-D SF-OPOs, as defined in [29]. For each time slice in the temporal profile of the pump, the coupled-mode equations that describe the simultaneously phasematched parametric generation and SFG processes in the nonlinear crystal of this class-D SF-OPO can be written as [29], [30]

$$
\begin{aligned}
\frac{\partial A_{1}}{\partial z} & =\frac{-j}{2 k_{1}}\left(\frac{\partial^{2}}{\partial x^{2}}+\frac{\partial^{2}}{\partial y^{2}}\right) A_{1}-j \frac{\omega_{1} d_{e a}}{n_{1} c} A_{3} A_{2}^{*} \\
\frac{\partial A_{2}}{\partial z} & =\frac{-j}{2 k_{2}}\left(\frac{\partial^{2}}{\partial x^{2}}+\frac{\partial^{2}}{\partial y^{2}}\right) A_{2}-j \frac{\omega_{2} d_{e a}}{n_{2} c} A_{3} A_{1}^{*}
\end{aligned}
$$




$$
\begin{aligned}
\frac{\partial A_{3}}{\partial z}= & \frac{-j}{2 k_{3}}\left(\frac{\partial^{2}}{\partial x^{2}}+\frac{\partial^{2}}{\partial y^{2}}\right) A_{3}-j \frac{\omega_{3} d_{e a}}{n_{3} c} A_{1} A_{2} \\
& -j \frac{\omega_{3} d_{e b}}{n_{3} c} A_{6} A_{4}^{*} \\
\frac{\partial A_{4}}{\partial z}= & \frac{-j}{2 k_{4}}\left(\frac{\partial^{2}}{\partial x^{2}}+\frac{\partial^{2}}{\partial y^{2}}\right) A_{4}-j \frac{\omega_{4} d_{e b}}{n_{4} c} A_{6} A_{3}^{*} \\
\frac{\partial A_{6}}{\partial z}= & \frac{-j}{2 k_{6}}\left(\frac{\partial^{2}}{\partial x^{2}}+\frac{\partial^{2}}{\partial y^{2}}\right) A_{6}-j \frac{\omega_{6} d_{e b}}{n_{6} c} A_{3} A_{4}
\end{aligned}
$$

where $A_{m}$ represent the complex field amplitudes of the optical electric field

$$
E_{m}(x, y, z, t)=\operatorname{Re}\left\{A_{m}(x, y, z, t) \exp \left[j\left(\omega_{m} t-k_{m} z\right)\right]\right\}
$$

and $m=1,2,3,4,6$ index the fields idler, signal, pump, rotated signal and sum-frequency, respectively. We note that $m=5$ is absent because parametric generation and SFG processes are coupled to each other through the pump, which is a field that is common to both processes. In the equations above, $d_{e a}$ and $d_{e b}$ are the effective nonlinear coefficients for parametric generation and SFG, respectively. Diffraction terms are included; however, beam walk-off terms are neglected since walk-off angles of the $p$-polarized beams are relatively small. Transmission losses experienced by the fields at the wavelengths of interest in KTA are negligible and, hence, not included.

Fourier transforming (1)-(5) into the spatial-frequency domain results in

$$
\begin{aligned}
\frac{\partial \tilde{A}_{1}}{\partial z}= & \frac{j 2 \pi^{2}}{k_{1}}\left(\nu_{x}^{2}+\nu_{y}^{2}\right) \tilde{A}_{1}-j \frac{\omega_{1} d_{e a}}{n_{1} c} \mathcal{F}\left(A_{3} A_{2}^{*}\right) \\
\frac{\partial \tilde{A}_{2}}{\partial z}= & \frac{j 2 \pi^{2}}{k_{2}}\left(\nu_{x}^{2}+\nu_{y}^{2}\right) \tilde{A}_{2}-j \frac{\omega_{2} d_{e a}}{n_{2} c} \mathcal{F}\left(A_{3} A_{1}^{*}\right) \\
\frac{\partial \tilde{A}_{3}}{\partial z}= & \frac{j 2 \pi^{2}}{k_{3}}\left(\nu_{x}^{2}+\nu_{y}^{2}\right) \tilde{A}_{3}-j \frac{\omega_{3} d_{e a}}{n_{3} c} \mathcal{F}\left(A_{1} A_{2}\right) \\
& -j \frac{\omega_{3} d_{e b}}{n_{3} c} \mathcal{F}\left(A_{6} A_{4}^{*}\right) \\
\frac{\partial \tilde{A}_{4}}{\partial z}= & \frac{j 2 \pi^{2}}{k_{4}}\left(\nu_{x}^{2}+\nu_{y}^{2}\right) \tilde{A}_{4}-j \frac{\omega_{4} d_{e b}}{n_{4} c} \mathcal{F}\left(A_{6} A_{3}^{*}\right) \\
\frac{\partial \tilde{A}_{6}}{\partial z}= & \frac{j 2 \pi^{2}}{k_{6}}\left(\nu_{x}^{2}+\nu_{y}^{2}\right) \tilde{A}_{6}-j \frac{\omega_{6} d_{e b}}{n_{6} c} \mathcal{F}\left(A_{3} A_{4}\right)
\end{aligned}
$$

where $\nu_{x}$ and $\nu_{y}$ are the spatial frequencies in $x$ and $y$ directions, respectively, $\tilde{A}_{m}\left(\nu_{x}, \nu_{y}, z, t\right)(m=1,2,3,4,6)$ are the Fourier transforms of the complex field amplitudes, and $\mathcal{F}$ Fourier-transforms a function $f(x, y, z, t)$ in the transverse dimension by calculating

$$
\iint_{-\infty}^{\infty} f(x, y, z, t) \exp \left[-j 2 \pi\left(\nu_{x} x+\nu_{y} y\right)\right] d x d y .
$$

Equations (7)-(11) are integrated using the Cash-Karp Runge-Kutta algorithm [33]. At the beginning of each $z$ step, $\tilde{A}_{m}\left(\nu_{x}, \nu_{y}, z, t\right)$ terms are inverse-Fourier-transformed to obtain $A_{m}(x, y, z, t)$ and the arguments of the Fourier transform function $\mathcal{F}$ in (7)-(11) are calculated.

In the double-pass configuration, the pump is reflected back into the cavity by M4. For the return pass, phase-matched parametric generation still occurs through the interaction of the reflected pump and resonating $p$-polarized signal; however, there is no SFG under the assumption that the $s$-polarized signal becomes completely depleted in the forward pass through the crystal. Equations (7)-(9) without the term due to SFG [the last term in (9)] are integrated in the return pass to account for parametric generation. The idler is again assumed to be zero at the starting location of the interaction (exit face of the crystal).

The $p$-polarized signal makes a double-pass through the $\lambda / 4$ plate and its polarization is rotated by an angle of $\alpha$. Hence, the updated complex field amplitudes of the $p$ - and $s$-polarized components after rotation are given by

$$
A_{2}^{\prime}=A_{2} \cos \alpha \text { and } A_{4}^{\prime}=A_{2} \sin \alpha .
$$

The Gaussian pulse fitted to the time profile of the pump laser which has a 17.8-ns pulse duration (FWHM) is discretized with 130 time slices each with a duration of the cavity round trip time of our SF-OPO which is $423 \mathrm{ps}$. The transverse spatial grid is typically $32 \times 32$ or $64 \times 64$ covering a computation area of $4.7 \times 4.7 \mathrm{~mm}$, which is the active CCD array size. When the output energy values calculated using a $128 \times 128$ grid are compared with those obtained using $32 \times 32$ and $64 \times 64$ grids, there are typically differences of $5 \%$ and $1 \%$, respectively, between the results.

Even when there is no polarization rotation $(\alpha=0), p$-polarized signal intensity (or energy) experiences a residual cavity loss. In the model we use the experimentally determined value of $2.5 \%$. Values that are used for the effective nonlinear coefficients $d_{e a}$ and $d_{e b}$ are 2.9 and $3.2 \mathrm{pm} / \mathrm{V}$, respectively. These values are calculated using the recently published data for the nonlinear tensor of KTA [34] with dispersion correction employing Miller's rule [35]. For the correction calculation, the Sellmeier coefficients given in [27] and [28] are used for parametric generation and SFG, respectively, at a propagation angle of $\theta=90^{\circ}$ and $\phi=30.1^{\circ}$, which is the simultaneous phase matching angle predicted by these refractive index data. It was observed that even a few percent change in one of these effective nonlinear coefficients results in typically a $10 \%$ change in the energy of the resultant sum-frequency pulse.

A seed power of $1 \mathrm{fW}$ is used for the signal in the model calculations. This value represents the spontaneous parametric fluorescence coupled into a cavity mode at full input energy [36]. It was observed that the model calculations are weakly dependent on the choice of the seed power. For instance, increasing the seed power by 3 and 6 orders of magnitude resulted in output energies which are larger than the one obtained at the seed power of $1 \mathrm{fW}$ by $\sim 4 \%$ and $\sim 8 \%$, respectively. This comparison was done for the double-pass SF-OPO operated at full energy and optimum $\alpha$.

\section{RESUlTS AND Discussion}

There is an optimum polarization rotation angle that maximizes the output pulse energy of the SF-OPO for a given pump input energy. When there is no polarization rotation (either the $\lambda / 4$ plate is removed from the cavity or its fast axis is aligned with either the $p$ - or $s$-polarization direction), the intracavity 


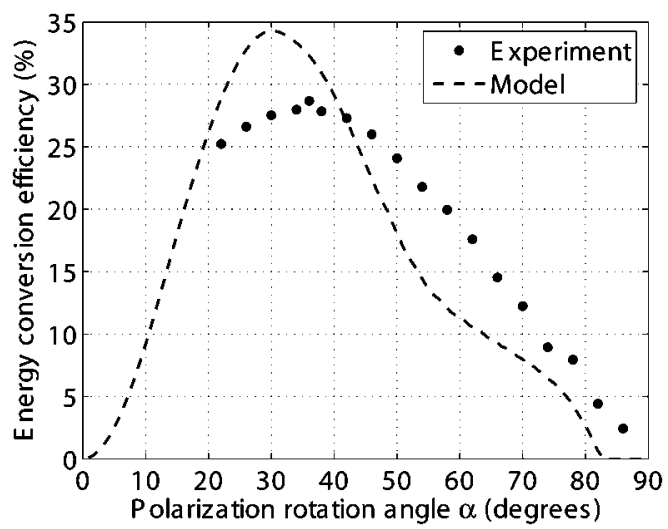

Fig. 2. Sum-frequency energy conversion efficiency as a function of the polarization rotation angle $\alpha$ for the double-pass configuration. Pump energy is held fixed at $20.4 \mathrm{~mJ}$. The predictions of our model are also shown.

signal beam does not have an $s$-polarized component, and, hence, there is no SFG. In this case, the residual cavity losses experienced by the signal beam is relatively small (approximately $2.5 \%$ ), resulting in a low OPO threshold and high intracavity signal intensity. As we start rotating the intracavity $\lambda / 4$ plate, hence, providing a polarization rotation, a portion of the $p$-polarized intracavity signal is coupled into $s$-polarization and SFG begins to take place. However, the effective linear cavity loss experienced by the resonating $p$-polarized signal, and, hence, the OPO threshold increase, as well. As a result, the SF-OPO falls below threshold at some polarization rotation angle above the optimum value. If the pump energy is increased, conversion to the signal wavelength increases and the optimum rotation angle increases as a result.

In this section, results are given as net values with the additional linear losses experienced by the pump beam at the input and by the sum-frequency beam at the output taken into account. Fig. 2 shows the 1064-nm-to-627-nm energy conversion efficiency as a function of the polarization rotation angle $\alpha$ at a fixed pump energy of $20.4 \mathrm{~mJ}$ for the double-pass configuration. The predictions of our model are also shown in the same figure. In the experiment, the smallest $\alpha$ is chosen to be $22^{\circ}$ to avoid damage to the KTA crystal. A peak conversion efficiency of $28.7 \%$ is obtained at the optimum polarization rotation angle of $36^{\circ}$. The SF-OPO falls below threshold at around $\alpha=90^{\circ}$. Our model predicts a peak conversion efficiency of $34.3 \%$ at $\alpha=30.1^{\circ}$.

The output sum-frequency energy at any input pump energy can easily be maximized by adjusting the polarization rotation angle. Fig. 3 shows the maximum energy conversion efficiency and the optimum polarization rotation angle as functions of the input pump energy for the double-pass configuration. Results of the model calculations are also shown in the same figure.

For the double-pass configuration, the achievable energy conversion efficiency at a given pump energy is much larger than that for the single-pass configuration, whereas the threshold energy is much smaller. Fig. 4(a) shows the output sum-frequency energy as functions of the pump energy for both double-pass and single-pass configurations at a fixed $\alpha$. The corresponding predictions of the model are also shown in the same subfigure. In the experiment, for the double-pass (single-pass) configuration

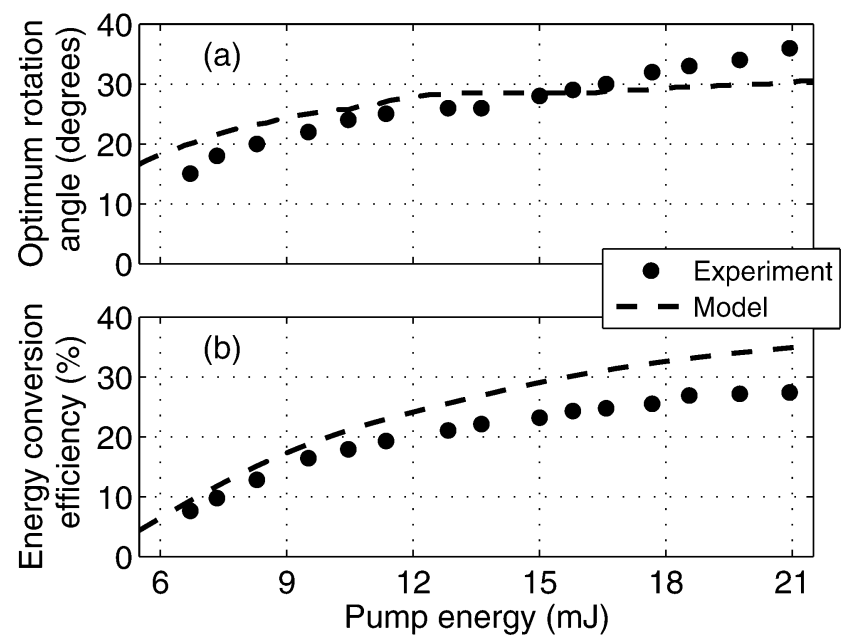

Fig. 3. (a) Optimum polarization rotation angle and (b) maximum energy conversion efficiency as functions of pump energy for the double-pass configuration. The predictions of the model are also shown.

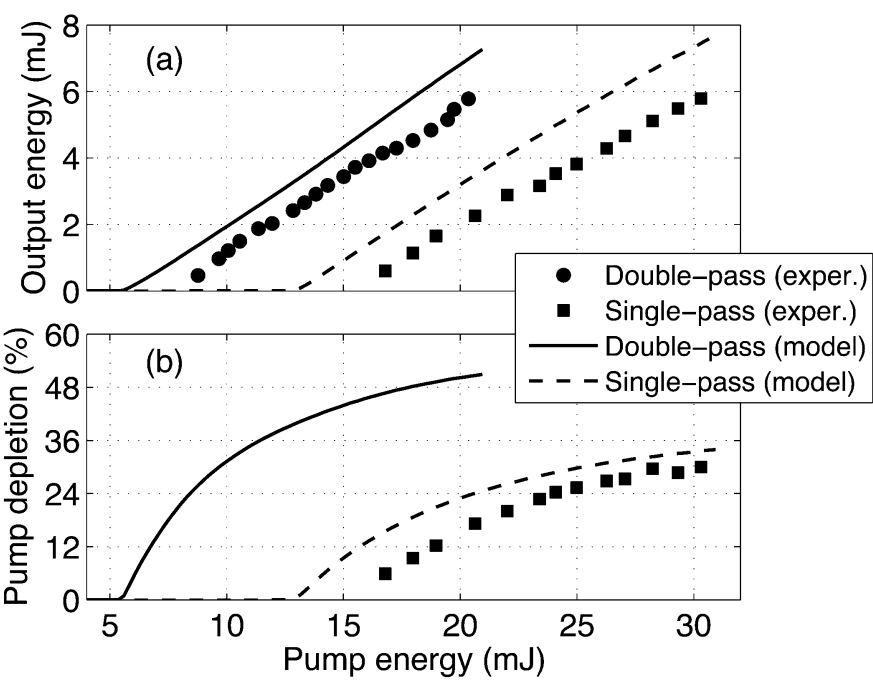

Fig. 4. (a) Output sum-frequency energy and (b) pump depletion as functions of pump energy for the double-pass and single-pass configurations. Polarization rotation angle is held fixed at $36^{\circ}$ and $38^{\circ}$ for the double-pass and single-pass configurations, respectively. The predictions of the model are also shown in the figure. For the model calculations, $\alpha$ is held fixed at the optimum values of $30.1^{\circ}$ and $29.4^{\circ}$ for the double-pass and single-pass configurations, respectively.

$\alpha$ is held fixed at $36^{\circ}\left(38^{\circ}\right)$, which is the optimum polarization rotation angle at the largest input energy of $20.4 \mathrm{~mJ}(30.3 \mathrm{~mJ})$. For the model calculations, $\alpha$ is held fixed at $30.1^{\circ}\left(29.4^{\circ}\right)$, which is calculated to be the optimum polarization rotation angle again at the largest input energy used in the double-pass (single-pass) configuration. In Fig. 4(b), the measured pump depletion is shown for the single-pass configuration; however, for the double-pass configuration, it could not be measured since the incident and return pump beams almost overlap each other. In the same subfigure, the calculated pump depletion for both double-pass and single-pass configurations is also shown.

For the double-pass configuration, a maximum of $5.8-\mathrm{mJ}$ sum-frequency energy is obtained at a pump energy of $20.3 \mathrm{~mJ}$, corresponding to $28.4 \%$ conversion efficiency. The threshold energy of the double-pass SF-OPO is only $7.7 \mathrm{~mJ}$. At the same 

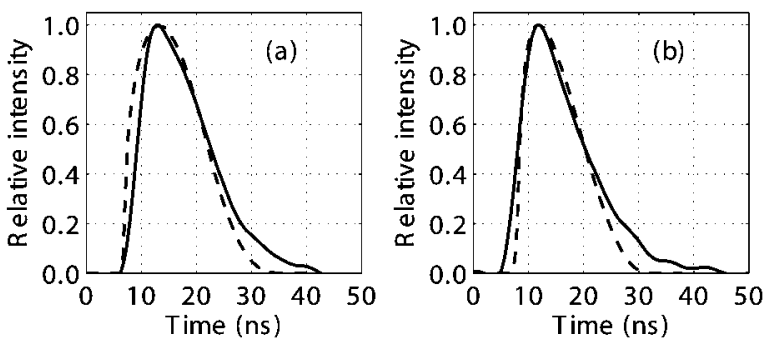

Fig. 5. Time profiles of the sum-frequency beam at full energy for (a) the double-pass and (b) single-pass configurations. Solid lines: measurement. Dashed lines: prediction of the model.

pump energy of $20.3 \mathrm{~mJ}$, our model predicts an output energy of $7 \mathrm{~mJ}$, corresponding to a conversion efficiency of $34.3 \%$ and a pump depletion of $50.4 \%$. The predicted threshold energy is $5.6 \mathrm{~mJ}$.

For the single-pass configuration, again a maximum of $5.8-\mathrm{mJ}$ sum-frequency energy is obtained at a pump energy of $30.3 \mathrm{~mJ}$, corresponding to $19.1 \%$ conversion efficiency and $30 \%$ pump depletion. The threshold energy of the single-pass $\mathrm{SF}-\mathrm{OPO}$ is as high as $15 \mathrm{~mJ}$. At the same pump energy of $30.3 \mathrm{~mJ}$, our model predicts an output energy of $7.5 \mathrm{~mJ}$, corresponding to a conversion efficiency of $24.6 \%$ and a pump depletion of $33.6 \%$. The predicted threshold energy is $12.9 \mathrm{~mJ}$.

Fig. 5 shows the time profiles of the sum-frequency beam measured at full energy (maximum input energy and optimum $\alpha$ ) for the double-pass and single-pass configurations. The time profiles predicted by the model at full energy for both configurations are also shown in the figure. For the double-pass configuration, the pulse duration (FWHM) of the sum-frequency beam is $14.2 \mathrm{~ns}$, whereas the value predicted by the model is $14.7 \mathrm{~ns}$. For the single-pass configuration, this value is $12.5 \mathrm{~ns}$, whereas it is predicted as $11.9 \mathrm{~ns}$ by the model.

The bandwidth of the pump beam is approximately $0.2 \mathrm{~nm}$. The spectra of the sum-frequency output measured at full energy peak at around $626.6 \mathrm{~nm}$ for both double-pass and single-pass configurations and they have bandwidths of 0.16 and $0.2 \mathrm{~nm}$, respectively. There is a slight spectral red-shift observed in the peak location $(\sim 0.04 \mathrm{~nm})$ for the double-pass configuration (with respect to that for the single-pass configuration), which is due to a slight shift in the signal wavelength resulting from the angle between the propagation axes of the incident and return pump beams $\left(0.2^{\circ}\right.$ in the KTA crystal). The output red beam of the double-pass SF-OPO at full energy is Gaussian-like with a diameter of $1.9 \mathrm{~mm}$ at the crystal exit and a divergence of 1.9 mrad.

Sellmeier coefficients given in [27] for parametric generation and those given in [28] for SFG closely predict the simultaneous phase matching angle determined experimentally. Fig. 6 shows the tuning curves of the signal wavelength calculated using the refractive index data given in [27] for parametric generation of the $p$-polarized signal from the $p$-polarized pump and refractive index data given in [28] for the sum-frequency interaction between the $s$-polarized signal and $p$-polarized pump $\left(\theta=90^{\circ}\right)$. The intersection of the two curves yields the simultaneous phase matching angle and occurs at $\phi=30.1^{\circ}$. We experimentally observed that SFG is most efficient for a propagation direction of $\theta=90^{\circ}$ and $\phi=30.8^{\circ}$, which is quite close to the predicted

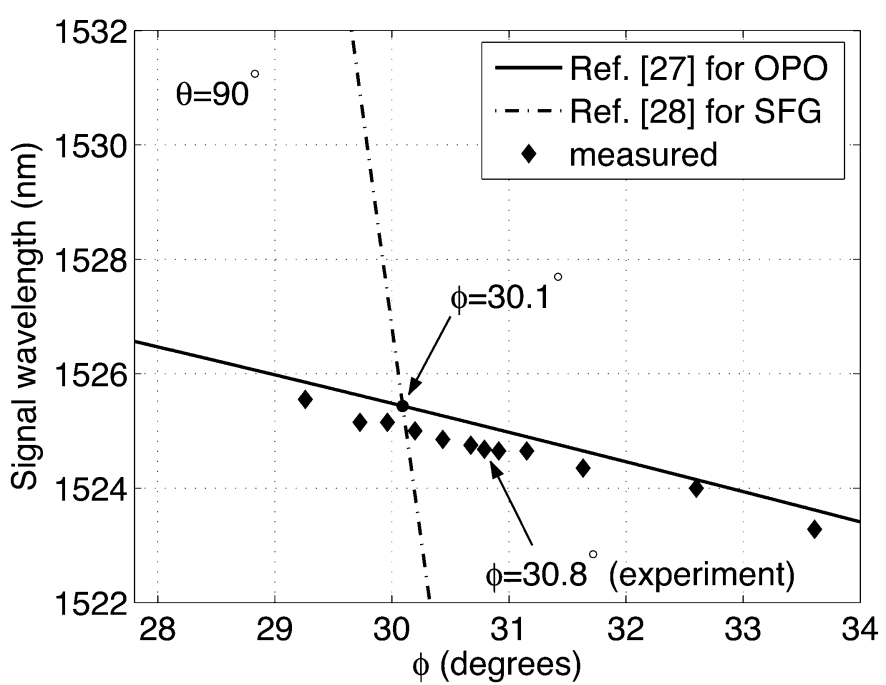

Fig. 6. Tuning curves of the signal wavelength calculated using the refractive index data given in [27] for parametric generation (signal is $p$-polarized) and refractive index data given in [28] for SFG (signal is $s$-polarized). Measured signal wavelengths are also shown. Signal wavelengths corresponding to the predicted and experimentally determined simultaneous phase-matching angles are indicated.

value. Signal wavelengths of the single-pass SF-OPO measured for various values of $\phi$ are also shown in Fig. 6. An output coupler that has a reflectivity of $85 \%$ at the signal wavelength was used for this measurement. Measured signal wavelengths are within $\pm 0.5 \mathrm{~nm}$ of those calculated using the Sellmeier coefficients given in [27].

\section{CONCLUSION}

We have demonstrated an upconversion OPO employing simultaneous phase matching which can convert the output of a $Q$-switched 1064-nm Nd:YAG laser into a red wavelength with upto $29 \%$ energy conversion efficiency. The device can be scaled for use with higher energy pump lasers by increasing the aperture size. Such scaling is not possible for OPOs based on periodically poled crystals due to the limited aperture size of these devices. Instead of slightly tilting the back-reflecting mirror M4 (see Fig. 1), a Faraday isolator can be placed between the pump laser and SF-OPO for preventing the coupling of the return pump beam into the pump laser cavity. It is further possible to shape our device into a physically small two-piece semi-monolithic configuration. In this case, mirrors M1, M2, and M4 can be coated onto the faces of the KTA crystal with its input face cut at an angle of $45^{\circ}$ with respect to the propagation direction in the crystal and a thin $\lambda / 4$ plate with one of its faces coated for high transmission and the other for high reflection at the signal wavelength (replacing M3) can attached onto the crystal, thereby minimizing the total cavity length and the energy threshold.

We developed a theoretical model for our device, which takes the temporal profiles of the beams and diffraction into account. Similar to the case for unseeded OPOs [30], this model predicts generally higher conversion efficiencies than the ones obtained in the experiment. This is to be expected since the model is for waves of monochromatic nature, hence, for narrow-bandwidth operation. Furthermore, in the model, we made no attempt to fit the predictions of the model to the data by adjusting any one of 
the physical parameters. For instance, the results are strongly dependent on the value of the effective nonlinear coefficients of the nonlinear processes $d_{e a}$ and $d_{e b}$. The values used in the model calculations were derived from those reported literature using Miller's scaling, which is only an approximation for taking the wavelength dependence of the effective nonlinear coefficient into account. Our model is the first model reported for SF-OPOs operating in the nanosecond regime and is in qualitative agreement with the experimental results.

\section{REFERENCES}

[1] G. J. Hall and A. I. Ferguson, "Generation of single-frequency radiation at 1064, 1319, and $659.5 \mathrm{~nm}$ with an all-solid-state, out-of-plane Nd:YAG ring laser," Opt. Lett., vol. 19, pp. 557-559, 1994.

[2] Y. Inoue, S. Konno, T. Kojima, and S. Fujikawa, "High-power red beam generation by frequency-doubling of a Nd:YAG laser," IEEE J. Quantum Electron., vol. 35, no. 11, pp. 1737-1740, Nov. 1999.

[3] Z. Sun, R. Li, Y. Bi, X. Yang, Y. Bo, Y. Zhang, G. Wang, W. Zhao, H. Zhang, W. Hou, D. Cui, and Z. Xu, "Generation of $11.5 \mathrm{~W}$ coherent red-light by intra-cavity frequency-doubling of a side-pumped Nd:YAG laser in a 4-cm LBO," Opt. Commun., vol. 241, pp. 167-172, 2004.

[4] J. R. Lincoln and A. I. Ferguson, "All-solid-state intracavity-doubled Nd:YLF laser producing $300 \mathrm{~mW}$ of 659-nm light," Opt. Lett., vol. 19, pp. 1213-1215, 1994.

[5] G. R. Morrison, M. Ebrahimzadeh, C. F. Rae, and M. H. Dunn, "Diodepumped, $Q$-switched $1.321 \mu \mathrm{m} \mathrm{Nd:YLF} \mathrm{laser} \mathrm{and} \mathrm{its} \mathrm{frequency} \mathrm{dou-}$ bling," Opt. Commun., vol. 118, pp. 55-60, 1995.

[6] A. Agnesi, A. Guandalini, and G. Reali, "Efficient 671-nm pump source by intracavity doubling of a diode-pumped Nd: $\mathrm{YVO}_{4}$ laser," $J$. Opt. Soc. Amer. B, vol. 19, pp. 1078-1082, 2002.

[7] H. Ogilvy, M. J. Withford, P. Dekker, and J. A. Piper, "Efficient diode double-end-pumped $\mathrm{Nd}: \mathrm{YVO}_{4}$ laser operating at 1342 nm," Opt. Exp., vol. 11, pp. 2411-2415, 2003.

[8] A. Agnesi, A. Guandalini, G. Reali, S. Dell'Acqua, and G. Piccinno, "High-brightness 2.4-W continuous-wave $\mathrm{Nd}: \mathrm{GdVO}_{4}$ laser at 670 nm," Opt. Lett., vol. 29, pp. 56-58, 2004.

[9] C. Du, S. Ruan, Y. Yu, and F. Zeng, "6-W diode-end-pumped $\mathrm{Nd}: \mathrm{GdVO}_{4} / \mathrm{LBO}$ quasi-continuous-wave red laser at $671 \mathrm{~nm}$," Opt. Exp., vol. 13, pp. 2013-2018, 2005.

[10] W. R. Bosenberg, L. K. Cheng, and C. L. Tang, "Ultraviolet optical parametric oscillation in $\beta-\mathrm{BaB}_{2} \mathrm{O}_{4}$," Appl. Phys. Lett., vol. 54, pp. 13-15, 1989.

[11] A. Fix, T. Schröder, and R. Wallenstein, "The optical parametric oscillators of beta-barium borate and lithium triborate: new sources of powerful tunable laser radiation in the ultraviolet, visible and near infrared," Laser Optoelektron., vol. 23, pp. 106-110, 1991.

[12] C. L. Tang, W. R. Bosenberg, T. Ukachi, R. J. Lane, and L. K. Cheng, "Optical parametric oscillators," Proc. IEEE, vol. 80, no. 3, pp. 365-374, Mar. 1992.

[13] D. E. Withers, G. Robertson, A. J. Henderson, Y. Tang, Y. Cui, W. Sibbett, B. D. Sinclair, and M. H. Dunn, "Comparison of lithium triborate and $\beta$-barium borate as nonlinear media for optical parametric oscillators," J. Opt. Soc. Amer. B, vol. 10, pp. 1737-1743, 1993.

[14] A. Fix, T. Schröder, R. Wallenstein, J. G. Haub, M. J. Johnson, and B. J. Orr, "Tunable $\beta$-barium borate optical parametric oscillator: operating characteristics with and without injection seeding," J. Opt. Soc. Amer. $B$, vol. 10, pp. 1744-1750, 1993.

[15] U. Bäder, J.-P. Meyn, J. Bartschke, T. Weber, A. Borsutzky, R. Wallenstein, R. G. Batchko, M. M. Fejer, and R. L. Byer, "Nanosecond periodically poled lithium niobate optical parametric generator pumped at $532 \mathrm{~nm}$ by a single-frequency passively $Q$-switched Nd:YAG laser," Opt. Lett., vol. 24, pp. 1608-1610, 1999.

[16] V. Pasiskevicius, H. Karlsson, F. Laurell, R. Butkus, V. Smilgevicius, and A. Piskarskas, "High-efficiency parametric oscillation and spectral control in the red spectral region with periodically poled $\mathrm{KTiOPO}_{4}$," Opt. Lett., vol. 26, pp. 710-712, 2001.

[17] R. A. Andrews, H. Rabin, and C. L. Tang, "Coupled parametric downconversion and upconversion with simultaneous phase matching," Phys. Rev. Lett., vol. 25, pp. 605-608, 1970.

[18] S. N. Zhu, Y. Y. Zhu, and N. B. Ming, "Quasi-phase-matched thirdharmonic generation in a quasi-periodic optical superlattice," Science, vol. 278, pp. 843-846, 1997.
[19] O. Pfister, J. S. Wells, L. Hollberg, L. Zink, D. A. Van Baak, M. D. Levenson, and W. R. Bosenberg, "Continuous-wave frequency tripling and quadrupling by simultaneous three-wave mixings in periodically poled crystals: application to a two-step 1.19-10.71- $\mu \mathrm{m}$ frequency bridge," Opt. Lett., vol. 22, pp. 1211-1213, 1997.

[20] K. G. Köprülü, T. Kartaloğlu, Y. Dikmelik, and O. Aytür, "Singlecrystal sum-frequency-generating optical parametric oscillator," J. Opt. Soc. Amer. B, vol. 16, pp. 1546-1552, 1999.

[21] X. P. Zhang, J. Hebling, J. Kuhl, W. W. Rühle, and H. Giessen, "Efficient intracavity generation of visible pulses in a femtosecond near-infrared optical parametric oscillator," Opt. Lett., vol. 26, pp. 2005-2007, 2001.

[22] K. Fradkin-Kashi, A. Arie, P. Urenski, and G. Rosenman, "Multiple nonlinear optical interactions with arbitrary wave vector differences," Phys. Rev. Lett., vol. 88, no. 023903, 2002.

[23] T. Kartaloğlu, Z. G. Figen, and O. Aytür, "Simultaneous phase matching of optical parametric oscillation and second-harmonic generation in aperiodically poled lithium niobate," J. Opt. Soc. Amer. $B$, vol. 20, pp. 343-350, 2003.

[24] T. Kartaloğlu and O. Aytür, "Femtosecond self-doubling optical parametric oscillator based on $\mathrm{KTiOAsO}_{4}$," IEEE J. Quantum Electron. vol. 39, no. 1, pp. 65-67, Jan. 2003.

[25] T. W. Ren, J. L. He, C. Zhang, S. N. Zhu, Y. Y. Zhu, and Y. Hang, "Simultaneous generation of three primary colours using aperiodically poled $\mathrm{LiTaO}_{3}$," J. Phys.: Condens. Matter, vol. 16, pp. 3289-3294, 2004.

[26] Z. G. Figen and O. Aytür, "Nanosecond sum-frequency generating optical parametric oscillator using simultaneous phase matching," Opt. Exp., vol. 13, pp. 4896-4902, 2005.

[27] D. L. Fenimore, K. L. Schepler, U. B. Ramabadran, and S. R McPherson, "Infrared corrected sellmeier coefficients for potassium titanyl arsenate," J. Opt. Soc. Amer. B, vol. 12, pp. 794-796, 1995.

[28] J.-P. Fève, B. Boulanger, O. Pacaud, I. Rousseau, B. Ménaert, G. Marnier, P. Villeval, C. Bonnin, G. M. Loiacono, and D. N. Loiacono, "Phase-matching measurements and sellmeier equations over the complete transparency range of $\mathrm{KTiOAsO}_{4}, \mathrm{RbTiOAsO}_{4}$, and $\mathrm{CsTiOAsO}_{4}$," J. Opt. Soc. Amer. B, vol. 17, pp. 775-780, 2000.

[29] Y. Dikmelik, G. Akgün, and O. Aytür, "Plane-wave dynamics of optical parametric oscillation with simultaneous sum-frequency generation," IEEE J. Quantum Electron., vol. 35, no. 6, pp. 897-912, Jun. 1999.

[30] A. V. Smith, W. J. Alford, T. D. Raymond, and M. S. Bowers, "Comparison of a numerical model with measured performance of a seeded, nanosecond KTP optical parametric oscillator," J. Opt. Soc. Amer. B, vol. 12, pp. 2253-2267, 1995.

[31] A. V. Smith, R. J. Gehr, and M. S. Bowers, "Numerical models of broad-bandwidth nanosecond optical parametric oscillators," J. Opt. Soc. Amer. B, vol. 16, pp. 609-619, 1999.

[32] G. Arisholm, "Quantum noise initiation and macroscopic fluctuations in optical parametric oscillators," J. Opt. Soc. Amer. B, vol. 16, pp. 117-127, 1999

[33] W. H. Press, B. P. Flannery, S. A. Teukolsky, and W. T. Vetterling, Numerical Recipies in C. New York: Cambridge Univ. Press, 1992.

[34] M. V. Pack, D. J. Armstrong, and A. V. Smith, "Measurement of the $\chi^{(2)}$ tensors of $\mathrm{KTiOPO}_{4}, \mathrm{KTiOAsO}_{4}, \mathrm{RbTiOPO}_{4}$, and $\mathrm{RbTiOAsO}_{4}$ crystals," Appl. Opt., vol. 43, pp. 3319-3323, 2004.

[35] D. A. Roberts, "Simplified characterization of uniaxial and biaxial nonlinear optical crystals: a plea for standardization of nomenclature and conventions," IEEE J. Quantum Electron., vol. 28, no. 10, pp. 2057-2074, Oct. 1992.

[36] R. L. Byer and S. E. Harris, "Power and bandwidth of spontaneous parametric emission," Phys. Rev., vol. 168, pp. 1064-1068, 1968.

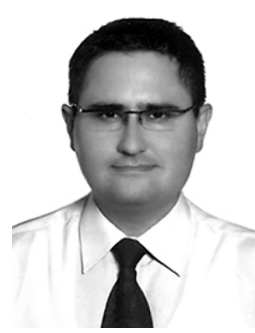

Ziya Gürkan Figen was born in Ankara, Turkey, in 1969. He received the B.S. degree from the Middle East Technical University, Ankara, in 1991, the M.S. degree from the University of Illinois at Urbana-Champaign (UIUC), Urbana, in 1994, and the Ph.D. degree from Bilkent University, Ankara, in 2005, all in electrical engineering.

He was a Researcher with the Department of Electrical Engineering, UIUC, from 1994 to 1998 . He is currently a Senior Scientist within the TÜBITAK UEKAE İLTAREN Research Group, Ankara. His main research interests include electrooptics, nonlinear frequency conversion of lasers, spectroscopy, and electromagnetics. 


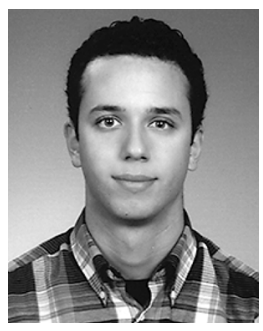

Ateş Yalabik was born in Ankara, Turkey, in 1982. $\mathrm{He}$ received the B.S. and M.S. degrees in electrical engineering from Bilkent University, Ankara, in 2003 and 2005 , respectively.

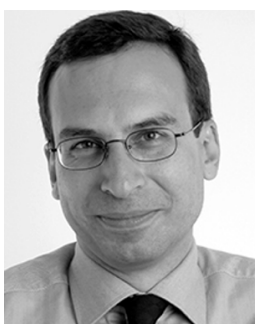

Orhan Aytür (M'94-SM'00) was born in Ankara, Turkey, in 1965. He received the B.S. degree in electrical engineering from Middle East Technical University, Ankara, in 1986, and the M.S. and Ph.D. degrees in electrical engineering from Northwestern University, Evanston, IL, in 1988 and 1991, respectively.

He was a Laser Scientist at Fibertek, Inc., Herndon, VA, and a Research Associate at the University of New Mexico, Albuquerque, from 1991 to 1992 . He joined the Department of Electrical Engineering, Bilkent University, Ankara, in 1993, where is presently a Professor. His main research interests include nonlinear frequency conversion of lasers, quantum optics, and high-performance photodetectors. 